\title{
Acidente por injeção medicamentosa no músculo deltoide: lesões locais e à distância, revisão de 32 casos
}

\author{
Intramuscular accident with drug injection in the deltoid muscle: local \\ and distant lesions, review of 32 cases
}

Fernando Luis Vieira Duque ${ }^{1}$, Carlos Alberto Araujo Chagas ${ }^{2}$

\section{Resumo}

Análise de 32 casos de acidentes por injeção, na maioria das vezes, de substâncias oleosas no músculo deltoide. Os acidentes caracterizaram-se por dor e reações tróficas locais. Em muitos casos, simultaneamente, ocorreram distúrbios isquêmicos nas regiões escapular, peitoral e, especialmente, na extremidade do membro. Presume-se que esse complexo lesional é gerado: 1) pela ação lesiva do medicamento nas terminações nervosas, nos tecidos moles e perivasais; e/ou 2) pela entrada fortuita da substância no interior dos vasos, produzindo embolia e/ou endotelite trombosante; e/ou 3) pelo despertar de fenômenos vasomotores.

As lesões da mão, geralmente, foram mais graves que as lesões deltoidianas, com a eventual perda de dedos, espontânea ou cirúrgica. Os diversos quadros clínicos do acidente foram semelhantes, mas algumas características lesionais permitiram identificar mecanismos fisiopatogênicos peculiares, o que tem significado conceitual e terapêutico.

Palavras-chave: Dermite livedoide, arterite cútis medicamentosa, embolia cútis medicamentosa, síndrome de Nicolau, síndrome de Freudenthal, acidente por injeção intramuscular, gangrena de mão.

\section{Introdução}

O advento da seringa de injeção hipodérmica em 1853 (Charles Gabriel Pravaz), logo seguida da fabricação da seringa inteiramente de vidro em 1869 (Georg Wilhelm Amathus Luer), permitiu a administração de medicamentos via parenteral. O novo processo fez sucesso, mas, imediatamente, surgiram as complicações. Em 1893, Fournier ${ }^{1}$ já anotava que as injeções mercuriais determinavam in situ "des sphacéles de veritables gangrenes locales". Segundo Nicolau', foi Welander ${ }^{3}$, em 1898, quem teria descrito o primeiro caso de necrose cutânea por injeção local. Em 1906, Nicolsky ${ }^{4}$ descreveu a gangrena de região

\begin{abstract}
Analysis of 32 cases of accidental injection of oily suspension (in most cases) into the deltoid muscle is reported. Pain and local ulcers characterized the accidents. In many cases, simultaneous ischemic disorders were observed in the scapular and pectoral regions and especially in the end of the upper limb. It is presumed that this complex lesion was caused by 1) the harmful action of the medication on nervous terminations, soft and perivascular tissues; and/or 2) occasional entrance of the substance into blood vessels, causing embolism and/or thrombotic endothelitis; and/or 3) vasomotor phenomena.

Hand injuries were generally more severe than injuries in the deltoid region, with occasional spontaneous or surgical loss of fingers. Clinical statuses were similar, though some lesions had features, which allowed identification of peculiar physiopathogenic mechanisms, with conceptual and therapeutic significance.
\end{abstract}

Keywords: Livedoid dermatitis, arteritis cutis medicamentosa, embolia cutis medicamentosa, Nicolau syndrome, Freudenthal syndrome, accidental intramuscular injection, gangrene of the hand.

glútea subsequente a injeções mercuriais e sugeriu a origem embólica oleosa da lesão. Em 1924, este tromboembolismo isquemiante foi bem caracterizado por Freudenthal ${ }^{5}$ em três casos de necrose de nádega provocada por injeção intramuscular de bismogenol no tratamento da sífilis. Foi Freudenthat ${ }^{5,6}$ quem denominou a nova entidade de embolia cútis medicamentosa. Um ano depois, Nicolau $^{2}$ descreveu um quadro clínico semelhante, também com injeção oleosa nos músculos glúteos, chamando a atenção para a discromia cutânea que ocorre na fase inicial do traumatismo (cútis livedoide) e para a gangrena subsequente. Nicolau distinguiu os casos provocados por embo-

\footnotetext{
1. Membro benemérito e fundador, SBACVRJ. Professor titular, Angiologia, Pontifícia Universidade Católica do Rio de Janeiro (PUCRJ), Rio de Janeiro, RJ. Chefe, Serviço de Angiologia, Hospital Geral da Santa Casa da Misericórdia do Rio de Janeiro, Rio de Janeiro, RJ. Ex-professor, Angiologia, Universidade Federal do Rio de Janeiro (UFRJ), Rio de Janeiro, RJ.

2. Membro, SBACVRJ. Angiologista, Serviço de Angiologia, Santa Casa da Misericórdia do Rio de Janeiro, Rio de Janeiro, RJ. Mestre, UFRJ, Rio de Janeiro, RJ Professor, Morfologia, Universidade Federal Fluminense (UFF), Rio de Janeiro, RJ. Professor, Anatomia, Universidade Gama Filho (UGF), Rio de Janeiro, RJ.
}

Não foram declarados conflitos de interesse associados à publicação deste artigo.

Artigo submetido em 25.03.09, aceito em 06.07.09.

J Vasc Bras. 2009;8(3):238-246.

Copyright (C) 2009 by Sociedade Brasileira de Angiologia e de Cirurgia Vascular 
lia oleosa dos casos de arterite trombosante gerados por medicamentos em veículo aquoso. Posteriormente, outros autores $^{7-9}$ se deram conta da existência de formas clínicas distintas e propuseram a classificação da doença em dois tipos: o exantema embólico benigno de Freudenthal e a dermite livedoide e gangrenosa de Nicolau. Esta classificação não foi afortunada e, desde então, a doença tem tido diferentes designações tais como embolia cútis medicamentosa, síndrome de Freudenthal, síndrome de Nicolau, dermite livedoide, dermite de Nicolau, dentre outras. Estes rótulos têm sido empregados indiscriminadamente para nomear todo e qualquer acidente no local de uma injeção intramuscular e, até mesmo, em casos de injeção hipodérmica com reação cáustica e/ou alérgica locais. No começo do século XX, foram descritos casos semelhantes, e alguns autores $^{10,11}$ os classificaram em diferentes quadros clíni$\cos$.

Embora a dermite de Nicolau fosse uma entidade mórbida já bem conhecida nos serviços de dermatologia do nosso meio ${ }^{12,13}$, ela só alcançou os campos angiológicos na época do nascimento da especialidade, em meados do século passado. Em 1947, Murgel e Regalla ${ }^{14}$ descreveram um caso de acidente por injeção intramuscular de penicilina benzatina no músculo deltoide e presumiram que as lesões cutâneas fossem secundárias à embolia, mas não associaram o quadro clínico à síndrome de Nicolau. Em 1948, pela primeira vez, deparamo-nos com este acidente no caso de um jovem acadêmico de medicina que tomava injeções de bismuto oleoso para o tratamento da sífilis: no momento da injeção do medicamento no músculo deltoide, ele sentiu forte dor local, logo seguida de palidez, dormência e frio na mão. Com os bloqueios anestésicos do gânglio estrelado simpático cervical, houve melhora dos distúrbios da mão. Entretanto, posteriormente, a lesão do deltoide ulcerou, deixou cicatriz retrátil, e houve necrose e mumificação de um dedo (Figura 1). O exame microscópico da pele da região deltoidea revelou a presença de material estranho nas pequenas artérias da região, sugerindo a entrada fortuita do medicamento na árvore arterial, ou seja, a natureza embólica da lesão. Calcados nos achados do exame local, presumimos que os distúrbios da mão tivessem a mesma origem embólica. A radiografia simples de mão, neste e em mais três casos, mostrou pequenos pontos opacos nos dedos tal como nos casos de Murgel ${ }^{14}$, Gammel $^{15}$ e Kimberley ${ }^{16}$. Esse achado foi considerado como

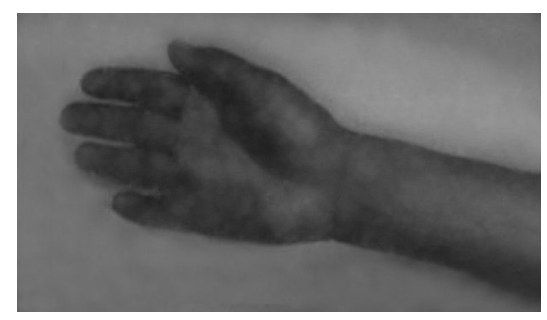

Figura 1 - Lesão isquêmica da mão após injeção intramuscular de bismuto oleoso no músculo deltoide (caso $n^{\circ} 1$, de 1948)

compatível com a presença de gotículas de sal de bismuto nas artérias. Em trabalho experimental em cadáveres feito na época ${ }^{17}$, constatamos que uma substância corante oleosa injetada nas artérias circunflexas do colo do úmero corria contracorrente para a artéria axilar e, daí, para as artérias da mão. Esses experimentos, por terem sido feitos em artérias exangues, não permitiram afirmar, categoricamente, a presença deste mecanismo patogênico nas lesões da mão dos pacientes. Em 1952, apresentamos seis casos desse acidente no I Congresso do Capítulo Latino-Americano de Angiologia, realizado em Buenos Aires ${ }^{17}$. Em todos os casos, além da lesão no local da injeção, havia, concomitantemente, distúrbios vasculares e tróficos na mão homolateral. À época, eram raras as publicações sobre este tipo de acidente ocorrendo na região deltoidiana e ainda mais raras, sobre as lesões em mão e dedos a ele associados. Havia relatos de casos semelhantes ocorridos na região glútea, com ou sem comprometimento do nervo ciático, mas com poucas referências a distúrbios circulatórios do pé $^{15,18}$. Nos anos seguintes, mais casos no membro superior foram apresentados em congressos ${ }^{19-21}$, mas sem ficar patente a etiopatogenia do processo. Em $1967^{22}$, foi constatada cirurgicamente a presença de substância oclusiva nas artérias do antebraço de um paciente que havia tido a lesão ombro-mão por injeção de penicilina procaína no músculo deltoide, confirmando a hipótese da embolia na gênese dos distúrbios vasculares e tróficos das mãos em pelo menos alguns desses casos. Os efeitos êmbolo-necróticos de substâncias oleosas injetadas diretamente na luz da artéria já eram conhecidos, tendo sido constatados em casos clínicos e comprovados experimentalmente, mas com certa variabilidade das lesões conforme a substância injetada. $\mathrm{Na}$ orelha de coelhos, a fenilbutazona produziu violenta inflamação seguida de necrose quando injetada na luz ou em torno da artéria ${ }^{23}$. Fenômeno semelhante ocorreu em experimentos em cães ${ }^{24}$. A injeção de penicilina 
benzatina com dipirona magnésica, responsável por um desses acidentes, não produziu lesões teciduais ou arteriais quando injetada experimentalmente em orelhas de coe$1 \operatorname{los}^{23}$. Entretanto, a injeção de penicilina benzatina ou de bismuto oleoso em patas de cães provocou lesões inflamatórias semelhantes às observadas nos casos clínicos ${ }^{24,25}$.

A síndrome da embolia cútis medicamentosa é uma entidade rara e ainda hoje pouco conhecida. Geralmente, é confundida com enfermidades semelhantes mais frequentes, que também são subsequentes a injeção de substâncias medicamentosas no músculo. Em nosso meio, nas décadas de 1940 e 1950, era generalizado o emprego de injeções de sais de bismuto e de mercúrio, em veículo oleoso, para o tratamento da sífilis. Na maioria das vezes, essas injeções eram aplicadas na região glútea mas, também, no músculo deltoide, onde é maior a possibilidade do medicamento ganhar o interior de um vaso. Nos anos seguintes, as injeções de penicilina benzatina e procainada passaram a ser as maiores responsáveis pelo acidente ${ }^{26}$. Com a diminuição do emprego de medicamentos oleosos ou em cristais por via muscular, houve redução da ocorrência desses eventos, e, nos anos recentes, os agentes agressores mais presentes tem sido os produtos em veículo aquoso, antigripais e anti-inflamatórios.

Nas últimas décadas, foram identificados muitos outros acidentes idênticos, aumentando também o número de casos relatados em publicações médicas e congressos ${ }^{27-44}$.

\section{Sintomas e sinais}

Na maioria das vezes, o acidente caracteriza-se pela dor e reação tecidual no local da injeção de aparecimento imediato ou tardio. A inflamação estéril tem evolução lenta, com eventual necrose, ulceração e cicatrização com fibrose retrátil.

Em alguns casos o acidente é mais grave e caracteriza-se pela dor intensa na hora e local da injeção, logo seguida da reação irritativa local. Geralmente, a pele em torno da punctura toma um aspecto esbranquiçado semelhante ao da palidez cadavérica; aos poucos, vão aparecendo placas cianóticas que se alternam com placas pálidas e formam um rendilhado de malhas grossas e irregulares com aspecto marmóreo livedoide. Mais tarde, as placas tornam-se escuras e, eventualmente, surgem vesículas com conteúdo sanguinolento. Aos poucos, as placas cianóticas vão ficando negras pela necrobiose isquêmica, e ins- tala-se a gangrena com extensão e profundidade variáveis. Quase sempre, a região deltoidea torna-se tumefeita e edematosa, com eventual formação de abscesso. Em dois dos nossos casos houve lesão tecidual do tipo fasciite necrosante. Em alguns casos ocorrem superinfecções fulminantes, gangrenas gasosas ou por anaeróbios, tal como é referido por alguns autores ${ }^{11,25,30,33,36,45,46}$.

Acredita-se que as lesões inflamatórias dos tecidos moles locais derivam da ação cáustica do produto, de sua concentração, de seu volume e, mais raramente, da ação mecânica da agulha. A agressão às terminações nervosas tem origem semelhante. Este complexo lesivo local pode ser acompanhado de um componente isquêmico-necrótico de maior ou menor intensidade e importância, gerado pelo comprometimento dos vasos - a lesão das artérias, arteríolas e veias ocorreria pela entrada acidental da substância injetada na luz do vaso ou pela ação cáustica sobre a adventícia, com subsequente inflamação da parede e trombose oclusiva. O produto medicamentoso oleoso, ao penetrar na artéria, gera êmbolos e oclui o lúmen - o medicamento em veículo aquoso, ao ganhar a luz do vaso arterial e/ou venoso, lesa o endotélio e provoca trombose secundária. As lesões das terminações nervosas somáticas e/ou perivasais desencadeiam reflexos simpáticos vasomotores com constrição vascular e agravamento da isquemia e/ou do enfartamento dos tecidos irrigados por esses vasos ${ }^{11}$.

Segundo é relatado, os acidentes ocorrem com o uso de uma vasta gama de remédios tais como corticosteroides, penicilinas, interferon alfa, metoclopramida, pirazolonas, anestésicos, noradrenalina, fenobutazona, diclofenaco, quimioterápicos, vitaminas e outros ${ }^{47}$. Os produtos oleosos mais citados são os bismúticos, os mercuriais, os balsâmicos e as penicilinas. O metocrilato plástico foi o responsável por um caso de necrose nasal após injeção em cirurgia por rinoplastia ${ }^{48}$.

Para Fernet ${ }^{11}$, salvo no caso de necrose tecidual, não haveria obstrução êmbolo-trombótica dos vasos locais: o espasmo vascular, provocado pela excitação da inervação simpática pelo medicamento, seria o responsável pelas formas benignas do acidente. A origem multifatorial da lesão local permitiu a Barthélemy ${ }^{10}$ postular a existência de quatro diferentes tipos de dermite livedoide: 1) o exantema embólico local; 2) a placa equimótica e flictenular; 3 ) a dermite livedoide e granulosa; 4) a gangrena profunda hi- 
podérmica e muscular. Ele não fez referências às lesões de mão que posteriormente observamos.

Algumas vezes a lesão do deltoide é acompanhada de distúrbios isquêmicos na extremidade do membro, que podem levar à necrose de dedos ou, até mesmo, da mão e do antebraço. Em quase todos os nossos pacientes, as alterações isquêmicas da mão regrediram, mas, em alguns, a lesão evoluiu para a necrose e mumificação da polpa ou de todo o dedo. Muitos autores citam casos em que foi necessária a amputação da mão ou mesmo do antebraço $^{3,7,12,22,25,28,49-52}$.

Os distúrbios isquêmico-necróticos à distância, que acompanham a lesão do ombro, geralmente constituem problema maior que a lesão do deltoide. Presume-se que a substância injetada penetre fortuitamente nas artérias circunflexas do colo do úmero, alcance a artéria axilar e daí caminhe para um de seus ramos, eventualmente condicionado pelas normais variações anatômicas da rede arterial $a x_{1 l a r}{ }^{53}$. Este é um dos mecanismos que explicaria a ocorrência de lesões nas regiões escapular, peitoral e, especialmente, na mão. No mesmo sentido, pode-se presumir que a entrada do material nas veias circunflexas gera flebite e enfartamento local; eventualmente, uma porção do medicamento pode fluir, via centrípeta, e provocar tromboflebite nos troncos venosos profundos. Nesta modalidade de acidente, o produto oleoso pode alcançar os pulmões e gerar quadros clínicos de embolia pulmonar². A lesão restrita aos nervos é mais rara e seria responsável por quadros nos quais predominam as manifestações sensitivas com pobre componente vascular.

Em quase todos os nossos casos, ocorreram distúrbios vasomotores e neurais locais ou à distância provocados pelos estímulos oriundos da excitação dos ramos simpáticos perivasais e/ou dos nervos mistos na região deltoidea ${ }^{23}$. Em alguns casos, o espasmo artério-arterial é o mais importante fator patogênico da síndrome e o principal alvo no seu tratamento.

A impotência funcional motora e/ou álgica do membro quase sempre esteve presente desde os primeiros momentos do acidente. Em quatro pacientes, houve a referência a desmaio ou tonteira no momento do acidente, sem outras manifestações sistêmicas.

O diagnóstico deste processo mórbido iatrogênico deltoidiano e do eventual comprometimento à distância é relativamente fácil e amplamente calcado na anamnese e no exame físico. A avaliação da presença e grau dos fenômenos vasomotores e oclusivos dos vasos da mão é bem realizada pela semiótica clínica desarmada, pela resposta ao bloqueio simpático e, especialmente, pela prova de hiperemia reativa provocada ${ }^{52}$. A radiografia simples de mão eventualmente revela a presença de microêmbolos de metais pesados nas artérias ${ }^{16,18,21}$. Certamente, o evento será melhor evidenciado pelos modernos processos de exame por imagem. Em nossos casos, o exame microscópico do tecido lesado só foi realizado para fins de investigação científica - a biópsia não é um processo semiotécnico necessário para o diagnóstico da síndrome, mesmo porque, em pacientes e em animais de experimentação, o exame histológico realizado tardiamente só revelou a presença de processo inflamatório inespecífico ${ }^{24,26,35-37,45,46,50,52-55}$. Os métodos semiotécnicos mais diferenciados estão indicados nos casos em que há oclusão orgânica de troncos arteriais e eventual indicação de reparação cirúrgica da artéria ocluída.

\section{Análise dos casos em conjunto}

Os 32 casos apresentados neste artigo não retratam a incidência de acidentes por injeção no músculo deltoide na população em geral, posto que o Hospital Geral da Santa Casa da Misericórdia do Rio de Janeiro não atende pessoas menores de idade, e nosso Serviço de Angiologia recebe pacientes encaminhados por outros serviços, não especializados. Outrossim, não foram computados no presente trabalho os casos com exame e/ou evolução incompletos. Além do mais, pacientes com lesões necróticas já instaladas geralmente são atendidos diretamente nos serviços de cirurgia.

O pequeno número de casos aqui estudados não permite realizar ilações estatísticas, mas é suficiente para a individualização de quadros clínicos - apesar da monótona similitude das lesões do ombro, e mesmo do complexo braço-mão, é possível identificar mecanismos etiopatogênicos diferentes, o que tem importância conceitual e terapêutica. Todos os pacientes são adultos jovens, e não houve diferença de incidência quanto ao sexo.

Em três pacientes surgiu dor intensa no momento da injeção, e houve reação inflamatória local com posterior necrose da pele (um caso) e cicatriz retrátil (dois casos). Em nenhum deles ocorreram distúrbios da mão, e nenhum 
melhorou com o bloqueio simpático. De todos os acidentes subsequentes às injeções intramusculares, este é o mais frequente, tanto na região deltoidea quanto na massa glútea. O pequeno número de casos deste tipo de lesão em nosso serviço talvez se deva ao fato deles serem relativamente benignos e tratados nos próprios locais do acidente, não havendo necessidade de um atendimento em serviço especializado. É provável que a lesão seja derivada da ação irritante e/ou alérgica do medicamento sobre os tecidos moles locais, especialmente no músculo e no hipoderma (miosite e paniculite cútis medicamentosa). Tem sido descrito que, eventualmente, ela toma a forma de paniculite factícia e evolui com formação de nódulos, perda de tecidos e depressão fibrosa cicatricial.

Em 2 pacientes a dor local foi intensa e se propagou por todo o membro e para o pescoço. As manifestações distróficas locais iniciais não foram marcantes, mas, dias depois, foram seguidas de atrofia de músculo e derma, com disfunção motora do deltoide. A distrofia cutânea local foi pequena, e não houve edema nem grandes distúrbios vasomotores do membro. O bloqueio anestésico do nervo simpático cervical não aliviou os sintomas. Mais tarde, um dos pacientes apresentou reflexo distrofia simpática no membro lesado ${ }^{20,56}$. A ausência de distúrbios circulatórios nesses dois casos permite supor que os vasos circunflexos não foram atingidos; também não se formaram estímulos vasomotores de monta. A substância irritante aquosa teria agredido somente as terminações nervosas da região, gerando a dor intensa imediata, assim como sua propagação, e a posterior atrofia do músculo deltoide (neurite cútis medicamentosa). Dali teria partido o estímulo que desencadeou o arco reflexo simpático anômalo. Nos dois pacientes restou grave prejuízo da movimentação do membro.

Após a injeção deltoidea de substância em veículo aquoso, três pacientes apresentaram pequena reação local com dor de média intensidade. Logo depois, o local da injeção ficou congesto e cianosado. Ao fim de algumas horas (12 horas em um paciente e na manhã seguinte nos outros dois), o membro superior edemaciou desde o ombro e a escápula até os dedos. Os movimentos do membro foram parcialmente prejudicados, houve dor difusa de média intensidade, coloração levemente azulada da pele de todo o membro e turgência das veias superficiais (Figura 2). O local da injeção estava tumefeito, sem sinais de necrose cutânea. Não foi feito bloqueio simpático. Com o uso de

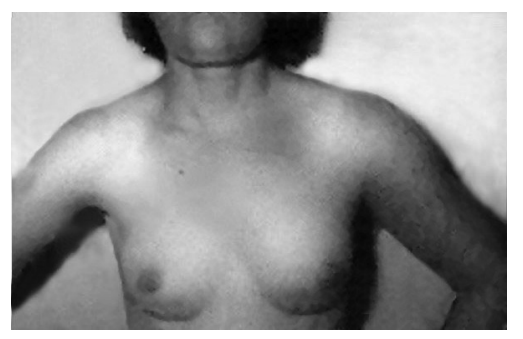

Figura 2 - Edema de membro superior após injeção de diclofenaco na massa deltoidea; flebite cútis medicamentosa e tromboflebite medicamentosa à distância

heparina e anticoagulante oral, houve melhora progressiva do quadro clínico. Semanas depois restava discreto edema de todo o membro e, em dois casos, pequena distrofia na região deltoidea. As mãos e dedos estavam normais. É de se presumir que o medicamento cáustico tenha entrado na(s) veia(s) circunflexa(s) e gerado disfunção endotelial e trombose com enfartamento local (flebite cútis medicamentosa). Outrossim, é possível que, ao fluir corrente acima, a substância irritante tenha alcançado a veia subclávia, provocando ali a tromboflebite profunda do membro (flebite medicamentosa).

Em seis pacientes, logo após a injeção de medicamento anti-inflamatório em veículo aquoso, houve dor intensa seguida de discromia livedoide na região deltoide, achado semelhante ao relatado por alguns autores. Em cinco casos, as lesões locais cursaram até necrose e esfacelo músculo-epidérmico. Três pacientes apresentaram distúrbios vasomotores nos dedos da mão e edema no ombro e na região escapular, que cederam espontaneamente (um caso) ou responderam ao bloqueio anestésico simpático (dois casos). Dois pacientes, atendidos tardiamente, não responderam ao bloqueio simpático e vieram a apresentar necrose em dedos. É possível que esta espécie de lesão seja oriunda da injeção do produto irritante na luz das artérias circunflexas, com subsequente disfunção endotelial, lesão da parede, trombose secundária e isquemia e necrose dos tecidos a jusante (arterite cútis medicamentosa) ${ }^{57}$. Os distúrbios circulatórios na mão parecem ser derivados de reflexos vasomotores gerados ao nível da injeção e/ou da ação do produto nos vasos da mão - o medicamento aí chegaria via artérias circunflexas do úmero e troncos arteriais do membro (arterite medicamentosa). Nestes casos, a dispersão do agente irritante na corrente sanguínea do membro talvez explique a pequena frequência dos distúrbios vasculares da mão. 
Em 18 pacientes, a substância medicamentosa injetada (bismuto, mercúrio, antigripal, penicilinas) estava em veículo oleoso. Em todos houve dor local importante no ato da injeção, logo seguida de dormência (nove casos) e de dormência e dor na mão e dedos (sete casos). Em dois pacientes, os sintomas na mão foram mínimos. Houve impotência funcional do membro em 13 casos. Em todos os pacientes o aspecto livedoide da pele em torno do local da injeção surgiu precocemente, logo seguido da formação de eritema, tumefação, vesículas com posterior necrose e ulceração (Figura 3). Dezesseis pacientes apresentaram isquemia de mão e/ou antebraço, com dor nos dedos, hipotermia, palidez e cianose de pele. Em dois pacientes, examinados no momento do acidente, os pulsos radial e ulnar estavam impalpáveis, mas se normalizaram após o bloqueio simpático cervical. Ocorreram distúrbios vasomotores e isquêmicos na região peitoral de dois pacientes e na região escapular de quatro deles. Os distúrbios vasomotores na mão responderam bem ao bloqueio anestésico da inervação simpática, que constituiu o principal tratamento
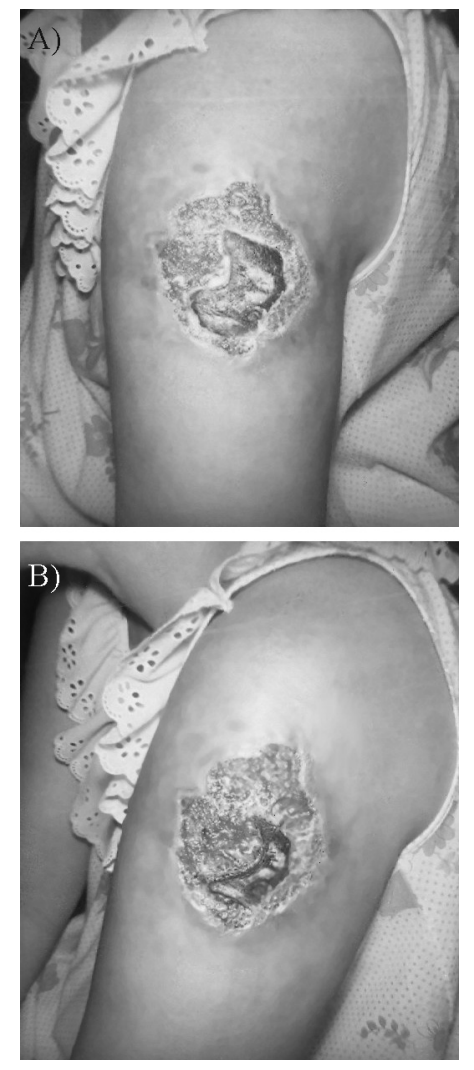

Figura 3 - Embolia cútis medicamentosa do deltoide por injeção de bismuto oleoso: A) placa necrótica inicial; B) lesão após duas semanas em quase todos os pacientes. Apesar de todos os esforços, ao fim de algum tempo de doença, todos os pacientes apresentavam lesão necrótica dos tecidos da região deltoidea. Oito tinham necrose em ponta de dedos (Figuras 4 e 5) e seis necessitaram ser submetidos à amputação cirúrgica das falanges de dois ou mais dedos (Figura 6).

Esta forma de acidente parece ser ocasionada: 1) pela entrada fortuita da substância oleosa na luz da(s) artéria(s) circunflexa(s), com oclusão arteríolo-arterial e bloqueio da

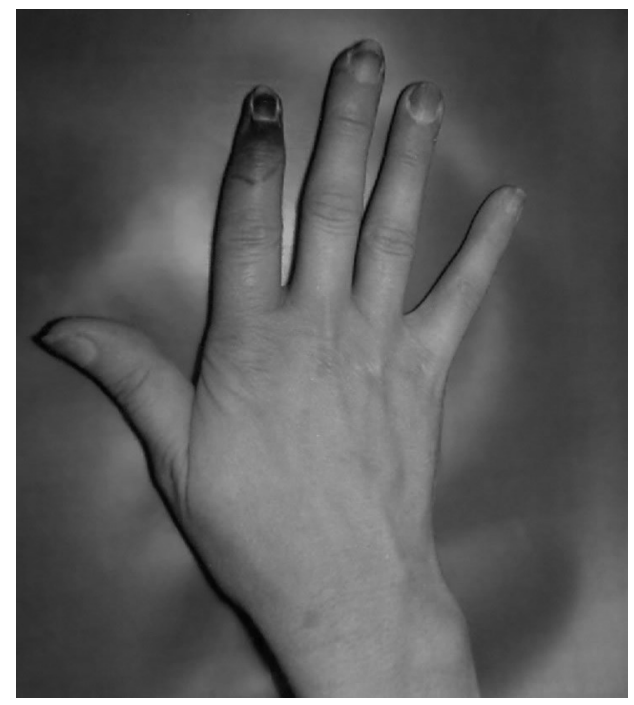

Figura 4 - Necrose de extremidade dos dedos após injeção intramuscular de bismuto no deltoide

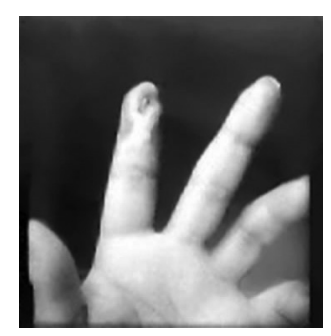

Figura 5 - Lesão residual da necrose de ponta de dedo desencadeada por embolia cútis medicamentosa no deltoide homolateral

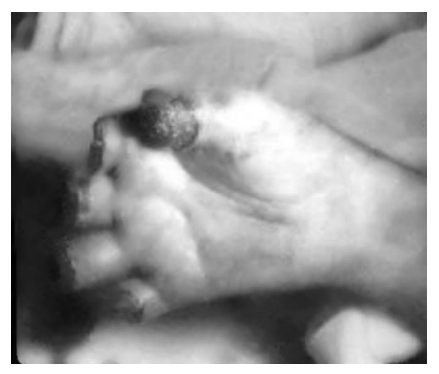

Figura 6 - Embolia cútis medicamentosa deltoideana e isquemia de mão. Amputação cirúrgica dos dedos necrosados 
circulação sanguínea a jusante (embolia cútis medicamentosa $)^{4,5,17}$; e 2) pelo fluir da substância oleosa nas artérias circunflexas, contracorrente, até alcançar a artéria axilar, correr pelos seus ramos escapulares e torácicos e, especialmente, pelos troncos arteriais da extremidade e ocluir os vasos distais (isquemia embólica) ${ }^{17}$. Os fenômenos vasomotores de braço e mão podem ter origem na excitação dos nervos periarteriais circunflexos ou na arterite embólica dos dedos. Este tipo de acidente tem curso mais grave que os outros acidentes talvez por dependerem tanto da endoteliopatia causada pelo medicamento quanto do tromboembolismo do veículo oleoso. A prevalência deste tipo de lesão em nossa casuística talvez se deva ao fato dos pacientes serem encaminhados ao serviço em face da presença e gravidade da lesão da mão.

De modo geral, os distúrbios isquêmicos da mão responderam bem aos bloqueios neurais simpático-cervicais. Em dois casos, a resposta ao bloqueio neural anestésico foi tão marcante que permitiu supor a presença do distúrbio vasomotor como principal agente da isquemia dos dedos, dependente ou não da embolia nas artérias da mão. Alguns autores ${ }^{58}$ acreditam que todas as lesões ocorrem por irritação dos nervos somáticos e/ou dos simpáticos perivasculares, desencadeando um reflexo vasomotor ganglionar, segundo postulou João Cid dos Santos ${ }^{55}$.

Na síndrome em estudo, não nos parece válido aceitar a presença de um mecanismo etiopatogênico único. A visão global dos casos agora apresentados permite identificar muitos sinais e sintomas comuns a todos eles, mas, também, constatar a existência de quadros clínicos diferentes (Figura 7). Em alguns casos, sobressai um fator etiopatogênico específico, mas na maioria deles, as manifestações parecem ser determinadas pela participação, em diferentes proporções, dos vários mecanismos patogênicos envolvidos no processo. A identificação dos mecanismos presentes em cada caso orienta a terapêutica.

\section{Tratamento}

Não há tratamento específico para a lesão da região deltoidea nem para a isquemia da mão. Entretanto, na maioria dos pacientes, é possível minimizar as consequências nocivas do acidente. As medidas terapêuticas aqui adotadas variaram conforme a apresentação e a evolução de cada caso. $\mathrm{O}$ combate à dor local foi realizado com medidas paliativas (anestésico cutâneo, frio, calor, etc.) sem

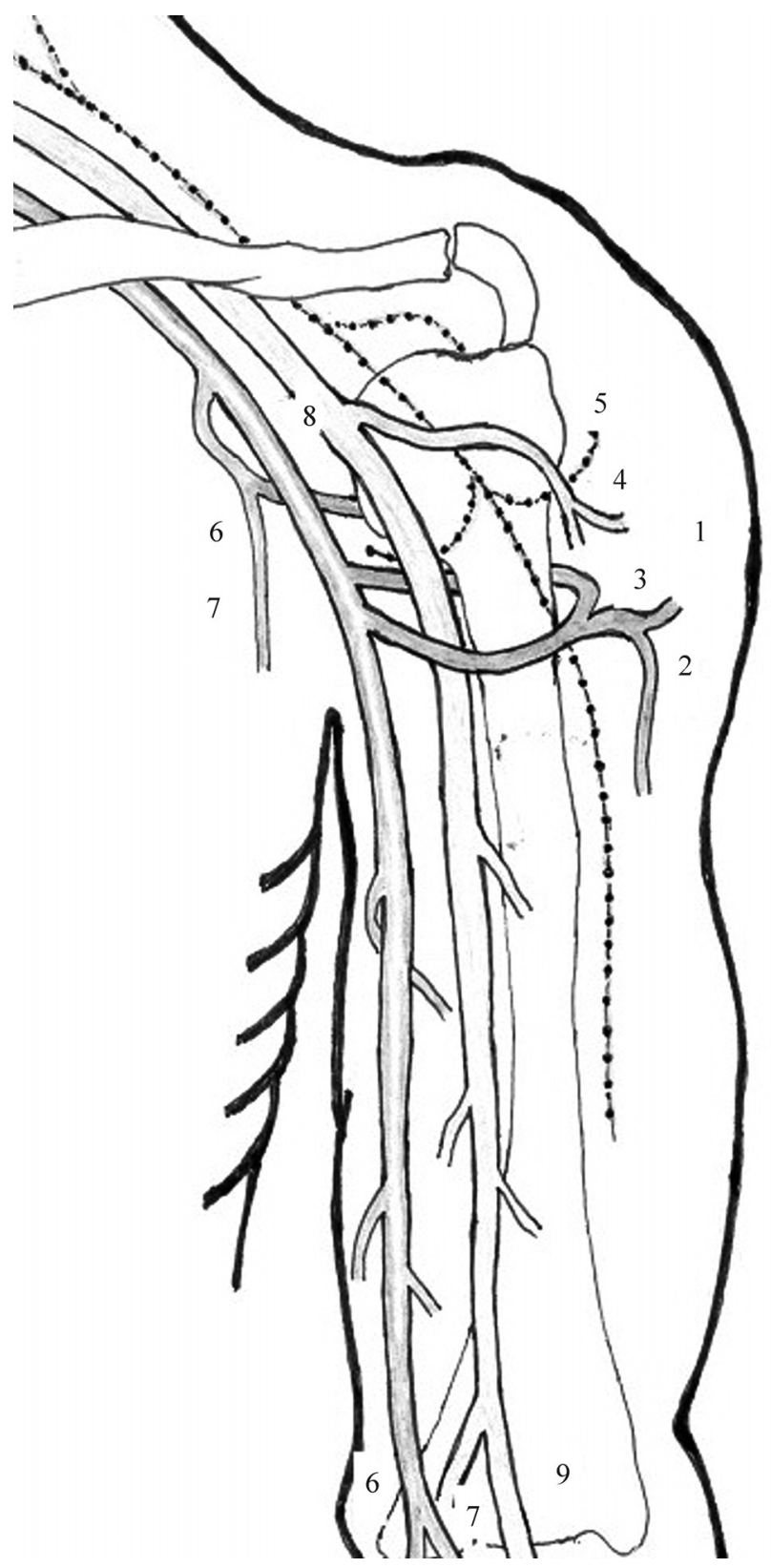

Figura 7 - Representação esquemática do complexo neurovascular deltoideano e eventuais sítios de acidentes medicamentosos. Lesões geradas por injeção intramuscular no músculo deltoide: 1) paniculite e miosite medicamentosas;2) embolia cútis medicamentosa; 3) arterite cútis medicamentosa; 4) flebite cútis medicamentosa; 5) neurite cútis medicamentosa; 6) embolia à distância; 7) arterite à distância; 8) flebite profunda; 9) vaso espasmo reflexo

grande sucesso; em casos raros, de dor mais intensa e/ou persistente, foi necessária a injeção local de substâncias anestésicas. O curso da ulceração do ombro foi acompanhado com os usuais cuidados terapêuticos locais (curativos, antissépticos, anestésicos) e com eventual execução de desbridamento cirúrgico. Em uns poucos casos, a infec- 
ção local tomou vulto e exigiu o emprego de antibiótico sistêmico. Os anticoagulantes foram administrados em pacientes com tromboflebite ou em aqueles com oclusão arterial digital orgânica que responderam mal ao bloqueio do nervo simpático. Em nenhum caso foi feito bloqueio do nervo simpático na vigência do uso de anticoagulantes.

Mesmo quando de pequena intensidade, o comprometimento vascular da mão foi tratado por meio de bloqueios anestésicos da cadeia simpático-cervical pela técnica de Souza Pereira, às vezes repetidos uma ou duas vezes por dia, durante os primeiros dias da doença. Em alguns casos mais rebeldes, o efeito vasodilatador foi potencializado com medicamentos orais e calor à distância. A lesão isquêmico-necrótica dos dedos foi manuseada da forma rotineira, tentando-se poupar o máximo de tecido e aguardando-se a delimitação da necrose antes de se realizar a amputação das falanges mumificadas. Não foi observado nenhum caso com oclusão de troncos arteriais do antebraço que exigisse medidas desobstrutivas e/ou reconstrutoras; da mesma forma, em nenhum dos pacientes houve gangrena de toda a mão ou do antebraço, tal como é descrito por muitos autores ${ }^{26,46}$. Na maioria dos pacientes restou alguma forma de distúrbio locomotor do membro que, quase sempre, regrediu com a fisioterapia e/ou com o passar do tempo.

Possivelmente, a pobreza de terapêutica específica para estes acidentes iatropatogênicos é um dos elementos que gera a relativa desobrigação do diagnóstico diferencial da síndrome e a monocórdica designação de síndrome de Nicolau para toda e qualquer reação anormal ocorrida com injeções intramusculares.

Parece claro que o melhor tratamento para esses acidentes é o profilático: evitar injeções de substâncias cáusticas e/ou oleosas no músculo deltoide ou observar o sítio adequado de aplicação e as demais recomendações básicas indicadas nos manuais de técnicas de enfermagem.

\section{Referências}

1. Fournier JA. Traitement de la syphilis. Paris: Rueff et Cie; 1893.

2. Nicolau SG. Dermite livedoide et gangréneuse de la fesse, consécutive aux injections intra-musculaires, dans la syphilis. A propos dun cas dembolie artérielle bismuthique. Annales des Maladies Vénériennes. 1925;20:321-39.

3. Cordeiro GC. Acidentes isquêmicos produzidos por injeções de substâncias medicamentosas [tese]. Rio de Janeiro: Universidade Federal do Rio de Janeiro; 1970.
4. Nicolsky P. Deux cas de gangrene de la fesse consecutive à une injection mercurielle. Bull Soc Fr Derm Syph. 1906; $17: 42$.

5. Freudenthal W. Lokales embolisches bismogenol-exanthem. Arch Derm Syphilol. 1924;147:155-60.

6. Freudenthal W. Medikamentöse Hautembolien (mit exanthem, blassenbildung, gangrän). Arch Derm Syphilol. 1927; 153:730-54.

7. Jeanselme E, Levy G, Huet L. Placard écchymotique et phlycténulair consécutif à une injection intra-fessière de Curalues. Bull Soc Fr Derm Syph. 1926;33:96-8.

8. Roederer J. Discussion sur les accidents provoqués par la medication bismuthique. Bull Soc Fr Derm Syph. 1929;7:1063-8.

9. Petges G, Joulia P. Au sujet des embolies artérielles bismutiques. Relation de cinq cas. Bull Soc Fr Derm Syph. 1930;7:1016-21.

10. Barthélemy R. Embolie artérielle par le bismuth liposolubile. Bull Soc Fr Derm Syph. 1930;37:1025-7.

11. Fernet P. La dermite livedoide et gangréneuse de Nicolau par embolie arterielle. Bull Soc Fr Derm Syph. 1930;7:1021-3.

12. Martins de Castro A. Embolie artérielle bismuthique avec lésions graves de la peau. Ann Derm Syph. 1929;VI:161-77.

13. Mesquita AP. Dermite livedoide de Nicolau e doença de Nicolas Favre. An Bras Derm Sifilogr. 1942;17:185-94.

14. Murgel L, Regalla J. Embolia arterial após injeção de preparado oleoso de bismuto. Hosp. 1947;31:875-8.

15. Gammel JA. Arterial embolism. An unusual complication following the intramuscular administration of Bismuth. J Am Med Assoc. 1927;88:998.

16. Kimberley LM. Accidental intra-arterial injection of bismuth in oil with demonstration of bismuth by $\mathrm{X}$ ray in the arteries of the penis. Amer J Syph. 1938;22:364-7.

17. Duque FLV. Embolia cútis medicamentosa de deltóide e isquemia de mão. In: I Congresso Latino-Americano de Angiologia; 1952; Buenos Aires, Argentina.

18. Gammel JA. Local accident following the intramuscular administration of salts of the heavy metals. Arch Derm Syphilol. 1928;18:210-23.

19. Duque FLV. Embolia cútis medicamentosa. Apresentação de oitos casos no membro superior, com lesão de mão. In: I Congresso Brasileiro de Angiologia; 1953; Belo Horizonte, Brasil.

20. Duque FLV. Quadros clínicos da reflexo distrofia simpática. In: II Congresso Latino-Americano de Angiologia; 1954; São Paulo, Brasil.

21. Duque FLV. Embolia cútis medicamentosa. In: V Congresso Latino-Americano de Angiologia; 1960; Rio de Janeiro, Brasil.

22. Perissé R, Fernandes AD, Monteiro A, Cortes JC, Meirelles ML, Azambuja M. Acidentes vasculares por injeção. J Bras Med. 1967;13:213-8.

23. Brachtel R, Meinertz T. Local skin necroses intramuscular after injection -Experimental animal studies. Arch Dermatol Res. 1977;258:281-8.

24. Cordeiro GC. Acidentes isquêmicos produzidos por injeções de substâncias medicamentosas [tese]. Rio de Janeiro: Universidade Federal do Rio de Janeiro; 1970. 
25. Cordeiro GC, Brum OF, Brito CJ, Jacques H, Arruda S. Necrose isquêmica do membro superior. Apresentação de um caso de arterite. In: XIV Congresso Brasileiro de Angiologia; 1967; São Paulo, Brasil.

26. Miranda MCC, Rosenfeld S, Oliveria SP. Reações adversas não alérgicas à suspensão Injetável de benzilpenicilina benzatina: uma revisão sistemática. J Vasc Bras. 2004;3;253-60.

27. Santler R, Ebner H, Mischer P. Drug induced cutaneous embolism. Hautarzt. 1972;23:530-4.

28. Ferreira AO, Portilho MA, Betino GA. Lesão isquêmica aguda iatrogênica do membro superior. Um caso. In: XXII Congresso Brasileiro de Angiologia; 1977; Minas Gerais, Brasil.

29. Stohr M, Dichgans J, Dorstelmann D. Ischaemic neuropathy of the lumbosacral plexus following intragluteal injection. J Neurol Neurosurg Psychiatry. 1980;43:489-94.

30. Mello NA, Eugenio AM, Castro S, Barros S, Duque FL. Arterites periféricas. In: XXIV Congresso Brasileiro de Angiologia; 1981; Rio de Janeiro, Brasil.

31. Vasconcellos CA, Velasco JA, Alves MM. Acidentes por injeção. Apresentação de 11 casos. In: XXIV Congresso Brasileiro de Angiologia; 1981; Rio de Janeiro, Brasil.

32. Thomaz JB, Baltar CA. Acidente isquêmico no membro superior produzido por injeção intramuscular de penicilina benzatina. Arq Bras Med. 1988;62:175-8.

33. Golcman B, Golcman R, Castro LG, Mizoguchi M. Necrose tecidual após injeção intramuscular de diclofenaco de sódio. An Bras Dermatol. 1991;66:65-9.

34. Faucher L, Marcoux D. What syndrome is this? Nicolau syndrome. Pediatr Dermatol. 1995;12:187-90.

35. Köhler LD, Schwedler S, Worret WI. Embolia cútis medicamentosa. Int J Dermatol. 1997;36:197-201.

36. Giovannetti $M$, et al. Necrose tecidual: efeito colateral do Voltaren ${ }^{\circledR}$ - relatos de caso e discussão da fisiopatologia. Rev Hosp Clin Fac Med Sao Paulo. 1993;48:39-42.

37. Yoshida WB, Rollo HA, Lastória S, et al. Isquemia de membro superior após injeção intramuscular de ampicilina benzatina e dipirona magnésica: relato de um caso. Cir Vasc Angiol. 1999; 15:109-15.

38. Luton K, Garcia C, Poletti E, Koester G. Nicolau Syndrome: three cases and review. Int J Dermatol. 2006;45:1326-8.

39. Saputo V, Bruni G. Nicolau syndrome caused by penicillin preparations: review of the literature in search for potential risk factors. Pediatr Med Chir. 1998;20:105-23.

40. Koklu E, Sarici SU, Altun D, Erdeve O. Nicolau syndrome induced by intramuscular vitamin $\mathrm{K}$ in a premature newborn. Eur J Pediatr. 2009; Mar 11. [Epub ahead of print].

41. Hamilton B, Fowler P, Galloway H, Popovic N. Nicolau syndrome in an athlete following intra-muscular diclofenac injection. Acta Orthop Belg. 2008;74:860-4.

42. De Sousa R, Dang A, Rataboli PV. Nicolau syndrome following intramuscular benzathine penicillin. J Postgrad Med. 2008;54:332-4.
43. Panariello L, Ayala F. Nicolau syndrome following intramuscular diclofenac injection: a case report. Dermatol Ther. 2008;21 Suppl 1:S10-2.

44. Selimoglu O, Basaran M, Ugurlucan M, Ogus TN. Rhabdomyolysis following accidental intra-arterial injection of local anesthetic. Angiology. 2009;60:120-1.

45. Mota J. Dermite livedoide e gangrenosa de Nicolau. J Sífilis Urol. 1936;6:171-7.

46. Santos OS. Gangrena de membros superiores por injeção de penicilina. Um caso. In: V Congresso do Capítulo Latino Americano de Angiologia; 1960; Rio de Janeiro, Brasil.

47. Erkek E, Tuncez F, Sanli C, et al. Nicolaus syndrome in a newborn caused by triple DTP (diphtheria-tetanus-petussis) vaccination. J Am Acad Dermatol. 2006;54:241-2.

48. Jaimovich CA. Necrose nasal pós-injeção de PMMA. Síndrome de Nicolau-Freudenthal. Curso Prático de Rinoplastia. $38^{\mathrm{a}}$ Enf. Rio de Janeiro: Santa Casa do Rio de Janeiro; 2006.

49. Silva W, Telles E, Marques S, Salles EA, Alcântara A, Carvalho A. Isquemia dos membros por injeções intramusculares. Considerações em torno de 8 casos. Angiopatias. 1965;5:4-13.

50. Albrecht KH, Littmann K, Richter HJ, Eigler FW. Tierexperimentelle Untersuchungen zur Embolia cutis medicamentosa. Langenbecks Archiv für Chirurgie. 1984;362:17-23.

51. Springel P, Duque FLV. Incidência das doenças vasculares periféricas em 700 pacientes examinados em ambulatório especializado. In: I Congresso Brasileiro de Angiologia; 1953; Belo Horizonte, Brasil.

52. Duque FLV. A prova da hiperemia reativa provocada. In: II Congresso Latino-Americano de Angiologia; 1954; São Paulo, Brasil e II Congresso Brasileiro de Angiologia; 1955; Salvador, Brasil.

53. Pulcinelli E, Borges FA, Branco FE, et al. Variações da artéria axilar e seus ramos. Cir Vasc Angiol. 2000;16:21-5.

54. Duque FLV. Embolia cútis medicamentosa. In: V Congresso Latino-Americano de Angiologia; 1960; Rio de Janeiro, Brasil.

55. Cid dos Santos J. Patologia geral das isquemias dos membros. Lisboa: Liv Luso-Hespanhola Ltda.; 1944.

56. Houli J, Duque FLV. Distrofia reflexa simpática. In: V Congresso do Capítulo Latino-Americano de Angiologia; 1960; Rio de Janeiro, Brasil.

57. Duque FLV, Bernardini EM. Arterite cutis medicamentosa. Progress on Angiology. In: Proceedings of the VIII International Congress Brazilian of Angiology. 1974;3:1077-81.

58. Bernstein SH, Hauser HB. Sensitivity reaction to intramuscular injection of benzathine penicillin. N Engl J Med. 1959;260:747-51.

Correspondência:

Dr. Carlos Alberto Araujo Chagas

Rua Conde de Bonfim, 100/401, Tijuca

CEP 20520-053 - Rio de Janeiro, RJ

Tel.: (21) 2565.7950, (21) 9797.8766

E-mail: carloschagas@sbacvrj.com.br; carloschagas@ cremerj.gov.br 